\title{
Harmonisation de méthodes de cartographie des végétations sous-marines
}

\author{
Claire NOEL ${ }^{1}$, Christophe VIALA ${ }^{1}$, Michel COQUET ${ }^{1}$, Gilles HERVE ${ }^{2}$, \\ Eric EMERY ${ }^{2}$. Didier SAUZADE ${ }^{2}$, Roger KANTIN $^{2}$, Sylvain COUDRAY $^{2}$ \\ ${ }^{1}$ SEMANTIC TS, 39 chemin de la Buge, 83110 SANARY s/Mer, France \\ noel@semantic-ts.fr \\ 2 IFREMER. Laboratoire côtier. Zone Portuaire de Brégaillon. BP 330. \\ 83507 LA SEYNE s/Mer. France Roger.Kantin@ifremer.fr
}

\section{Résumé :}

Cet article présente les résultats de la comparaison de la méthode DIVA (Détection \& Inspection Verticale Acoustique), développée par SEMANTIC TS, avec les méthodes optiques et acoustiques mises en œuvre par l'IFREMER dans le cadre du programme INTERREG IIIB / Posidonia (www.ifremer.fr/posidonia/) sur deux sites ateliers : celui des Embiez à l'Ouest de Toulon et celui de Saint Raphaël. La méthode DIVA est particulièrement performante lorsqu'elle intervient en tant que capteur supplémentaire des méthodes surfaciques habituellement mises en œuvre par l'IFREMER.

\begin{abstract}
:
This paper presents research tasks conducted by SEMANTIC TS and IFREMER, in order to compare their underwater vegetation mapping methods. New DIVA method for detecting and characterizing vegetation on the seabed using the acoustic response from a conventional single beam echo sounder, and methods used by IFREMER and based on towed video and side scan sonar, are used and compared on the same areas studied in the context of INTERREG IIIB / Posidonia program (www.ifremer.fr/posidonia) $)$.
\end{abstract}

\section{Mots-clés :}

Cartographie végétation sous-marine - Acoustique sous-marine - Classification Sonar latéral - Echosondeur, Posidonie 


\section{$1 \quad$ Introduction}

Les méthodes de cartographie des herbiers font appel à des techniques aériennes, acoustiques, optiques, ainsi qu'à des plongées. En ce qui concerne les méthodes optiques, l'IFREMER a mis au point un dispositif de vidéo remorquée dédié à la cartographie des végétations sous-marines appelé " MOBIDIC » (Module d'Observation des Biocénoses par Imagerie Digitale pour le Côtier). Ce dispositif est complémentaire des méthodes acoustiques utilisant un sonar latéral utilisées habituellement par l'IFREMER (EDGETECH $110 \mathrm{KHz}$ ).

En matière de caractérisation acoustique des fonds, sont disponibles sur le marché plusieurs systèmes, un des plus connus étant le système RoxAnn de la société écossaise Stenmar Micro Systems Ltd..

De son côté, la société SEMANTIC TS mène depuis 2004 des travaux de recherche dans le domaine particulier de la cartographie acoustique des herbiers de posidonies, pour lesquels elle développe une méthode de détection acoustique à la verticale du navire particulièrement innovante. Cette méthode est appelée DIVA (Détection \& Inspection Verticale Acoustique). Afin de mieux qualifier les performances de cette méthode, elle met en œuvre différents capteurs acoustiques : sonars latéraux et sondeurs bathymétrique multi-faisceaux (KLEIN 5000 et GEOSWATH) et echosondeur (SIMRAD ES60). Elle travaille de plus sur la fusion des données acoustiques en provenance de ces capteurs.

Cet article présente dans une première partie les résultats de l'harmonisation de la méthode DIVA avec la méthode vidéo remorquée de l'IFREMER sur la zone de la Vaille située à proximité de l'archipel des Embiez. Il compare ensuite les résultats de la méthode DIVA avec celles mises en œuvre dans le cadre du programme INTERREG IIIB / Posidonia (www.ifremer.fr/posidonia/) sur deux zones d'étude à St Raphaël.

Par rapport au système RoxAnn, qui équipe notamment la vedette « Haliotis » de l'Ifremer, essentiellement ciblé sur la caractérisation des substrats, la méthode DIVA a été conçue pour travailler sur la couverture végétale et notamment l'herbier de Posidonie en Méditerranée. Le traitement (modulable) de son signal permet notamment de rechercher la meilleure caractérisation du signal recueilli. Le système RoxAnn, quant à lui, ne travaille pas dans le même spectre de retour. Il traite en temps réel le signal de retour et fournit de manière formatée à l'avance deux indices (un premier indice de rugosité E1 et un second indice de dureté E2), ce qui permet de classer automatiquement la nature des fonds en fonction de leur dureté (sédiment vaseux et lisse et sédiment grossier et rugueux par exemple). 


\section{$2 \quad$ Méthodes mises en oeuvre}

\subsection{Méthode DIVA (Détection \& Inspection Verticale Acoustique)}

La méthode de reconnaissance acoustique DIVA fonctionne de la manière suivante : le navire instrumenté se déplace sur la zone à lever; comme l'illustre la figure 1a; un PC installé à bord, récupère les informations en provenance des divers instruments de mesure, c'est-à-dire :

- la position (latitude, longitude et élévation de la mer) indiquée par un GPS différentiel,

- la réponse impulsionnelle du signal sonore réverbéré par le fond fournie par l'échosondeur.

Le PC traite les informations et, à une latitude et une longitude, il associe les informations caractéristiques de la végétation. SEMANTIC TS dispose sur son navire instrumenté d'un sondeur acoustique monofaisceau qui permet ce type de fonctionnement. Ce sondeur (modèle Simrad ES60) a déjà été utilisé par des équipes de l'INRA pour la caractérisation d'espèces de poissons (travaux similaires) à partir du signal acoustique réverbéré par les bancs de poissons (LURTON 2002). Le système analyse et traite la réponse impulsionnelle et en déduit les caractéristiques de la végétation fixée sur le fond sous-marin. Sur le sondeur utilisé, la réponse acoustique du sondeur est différente selon que le son se réfléchit sur une zone couverte de végétation ou non, comme l'illustre la Figure $1 \mathrm{~b}$ présentant deux "échos" (niveau sonore en fonction de la profondeur) représentatifs d'un fond de sable et d'un fond de posidonies. (NOEL et al 2005, VIALA et al 2006, VIALA et al 2007, VIALA et al 2008).

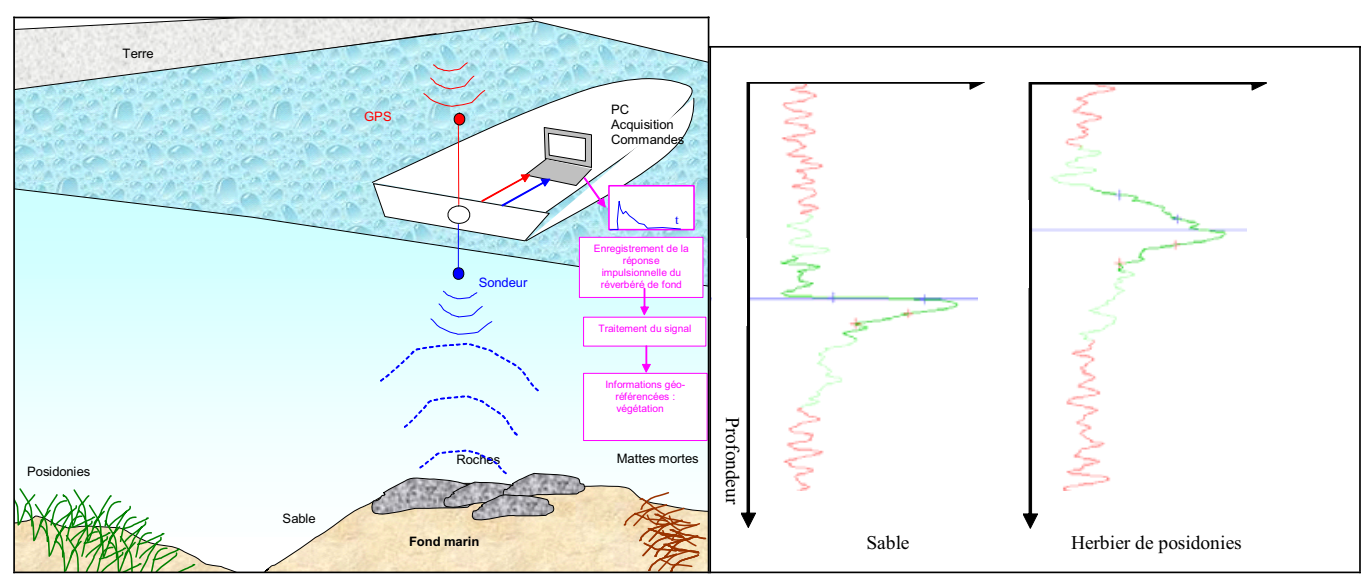

Figure 1. a) Principe de la méthode DIVA b) Echos représentatif du sable et de la posidonie

Les signaux sonores présentent donc une quantité d'énergie, avant le pic relatif à l'interface eau/fond, d'autant plus élevée que la biomasse végétale est importante. 
Les caractéristiques de la méthode DIVA sont les suivantes :

- Précision : $1 \mathrm{~m}$ à $10 \mathrm{~m}$ de profondeur, $3 \mathrm{~m}$ à $25 \mathrm{~m}$

- Récurrence d'acquisition : 5 par seconde (recouvrement des taches au sol)

- Vitesse acquisition : $7 \mathrm{Nd}$

- Linéaire couvert : $100 \mathrm{~km} / \mathrm{jour}$

\subsection{Système MOBIDIC}

Le dispositif vidéo MOBIDIC HD (Module d'Observation des Biocénoses par Imagerie Digitalisée pour le Côtier à Haute Définition) mis au point par l'IFREMER a été utilisé en parallèle à la méthode DIVA. Ce dispositif se compose des éléments suivants :

- un caméscope numérique haute définition de marque Sony, modèle HDR HC1, placé à l'intérieur d'un caisson cylindrique transparent et étanche clos par un hublot sphérique permettant une vision grand angle, pouvant résister aux pressions rencontrées à $6000 \mathrm{~m}$.

- un altimètre, placé dans l'enceinte du boîtier, permettant de situer la position du dispositif par rapport au fond.

- Avec le transformateur alimentant le caméscope, l'ensemble atteint $3,9 \mathrm{~kg}$ connecteurs compris.

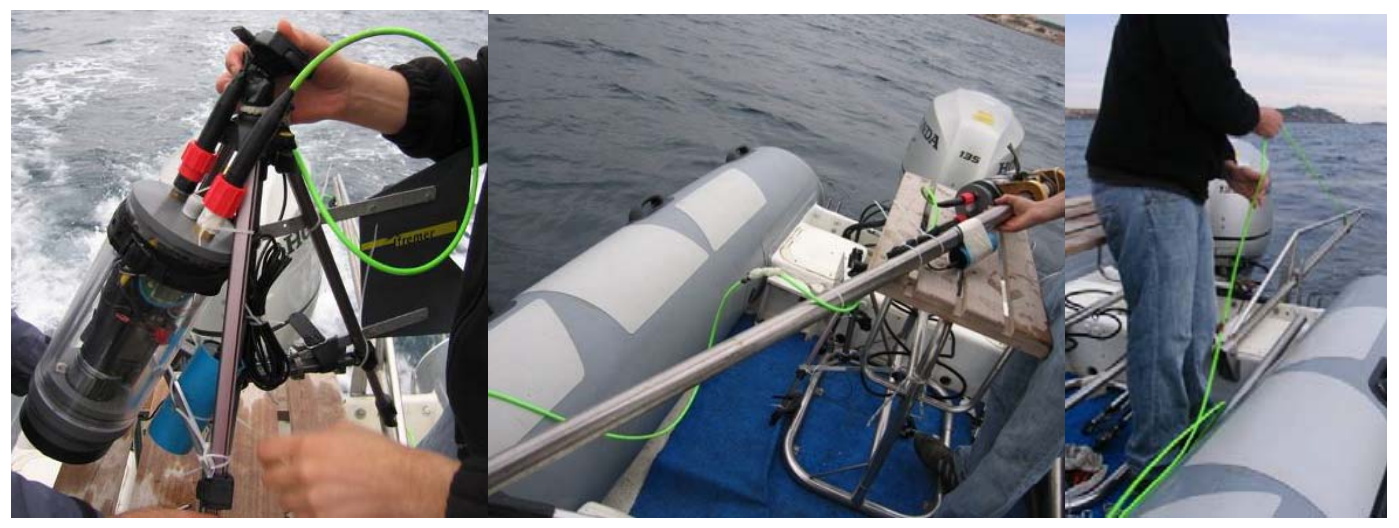

Figure 2 : De gauche à droite : caisson MOBIDIC-Perche support - Tangon

Deux méthodes ont été testées pour entraîner le caisson d'enregistrement vidéo :

- un prototype de fixation rigide pour le système MOBIDIC (perche de $4 \mathrm{~m}$ de long fixée dans le puit de mesures du navire SEMANTIC) qui maintient le caisson orienté vers le fond à environ 1,50 m de l'embarcation.

- le tractage du système MOBIDIC HD derrière le bateau par un câble électroporteur d'une longueur d'environ $30 \mathrm{~m}$ (100 m en eaux plus profondes) 
Les caractéristiques du système MOBIDIC sont les suivantes :

- Précision moyenne : $5 \mathrm{~m}$ à $1.5 \mathrm{Nd}$ (précision du GPS en point fixe)

- Récurrence d'acquisition : 1 par 2 secondes

- Vitesse acquisition : $1.5 \mathrm{Nd}$

- Linéaire couvert : 5 à $10 \mathrm{~km} / \mathrm{jour}$

\subsection{Sonar latéral mis en œuvre par l'IFREMER}

Le sonar latéral mis en oeuvre par l'IFREMER est le modèle DF1000 de la société Edgetech. Il insonifie les fonds en bi-fréquences à 100 et $380 \mathrm{kHz}$. La largeur du faisceau est de $50^{\circ}$ transversalement et de $1,2^{\circ}$ à $100 \mathrm{kHz}$ ou $0,5^{\circ}$ à 380 $\mathrm{kHz}$ dans la direction longitudinale, selon la fréquence de transmission choisie. Pour des raisons de sécurité du fait du tractage par câble électro-porteur $d u$ poisson et de la dégradation de la qualité des données, ce type de dispositif convient de préférence à la prospection des fonds de plus de $10 \mathrm{~m}$.

Les caractéristiques moyennes d'un sonar latéral tracté sont les suivantes :

- Précision moyenne : 5 à $10 \mathrm{~m}$ selon la longueur du câble et la rectitude de la trajectoire

- Récurrence d'acquisition : 1 à 5 par seconde

- Vitesse acquisition : $5 \mathrm{Nd}$

- Surface couverte : $100 \mathrm{~m}$ à $200 \mathrm{~m}$ de largeur de fauchée selon la fréquence utilisée avec un linéaire couvert de $70 \mathrm{~km} /$ jour. 


\section{Résultat de l'harmonisation}

\subsection{Méthode DIVA / Système MOBIDIC}

Les données acquises par l'échosondeur ont été dépouillées et traitées avec la méthode de détection acoustique DIVA. L'IFREMER et SEMANTIC TS ont analysé de façon conjointe les résultats de ces expérimentations afin de définir les performances de la méthode acoustique. Des images de faciès homogènes et des images au niveau des transitions entre faciès distincts, obtenues grâce à la vidéo remorquée, ont été analysées («rejeu»). Leur géoréférencement a été synchronisé sur l'heure GPS du navire SEMANTIC. Les images de la figure 3 montrent deux instantanés, issus de la vidéo, et correspondant à des transitions entre faciès benthiques distincts, positionnés sur l'imagerie du fond. La grande précision du positionnement de l'ensemble des données permet de constater que le type de fond visualisé sur chaque photographie, l'interprétation de la mosaïque, ainsi que les calculs effectués avec la méthode DIVA sont cohérents.. Les essais ont montré par ailleurs l'intérêt de la fixation de type perche pour un meilleur enregistrement vidéo dans des profondeurs inférieures à $10 \mathrm{~m}$ (augmentation de la précision du géoréférencement des images ainsi que la vitesse d'acquisition des données).

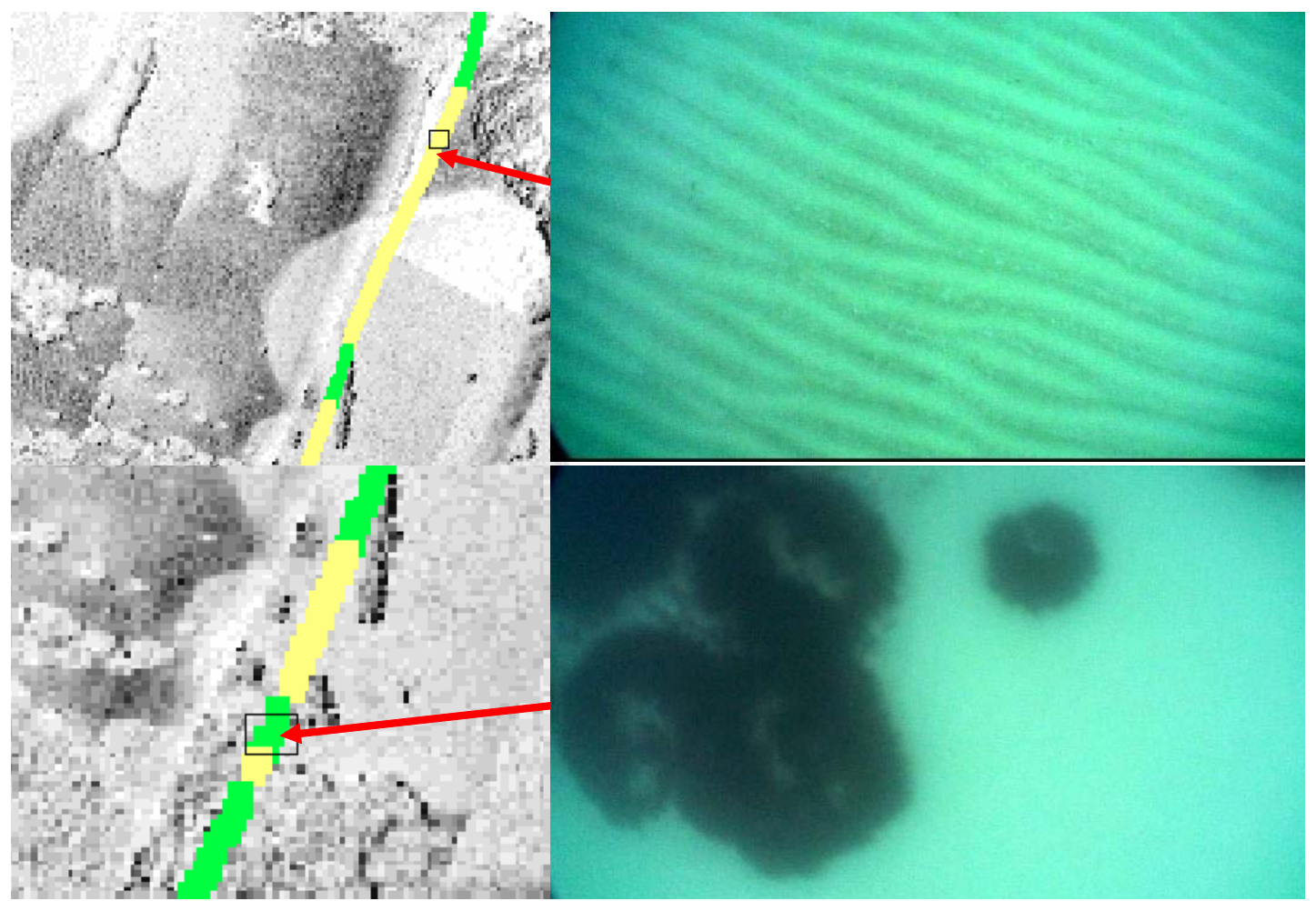

Figure 3: A gauche mosaïque sonal \& résultats de DIVA (Jaune/ Sable Vert/Posidonie) A droite : Vue MOBIDIC. En haut : Ridules de sable grossier. En bas : Taches d'herbiers sur sable fin 


\subsection{Méthode DIVA / Sonar latéral}

Les résultats obtenus avec la méthode DIVA sont présentés à la figure 4, en superposition de la mosaïque obtenue avec le sonar latéral Edgetech DF 1000, à l'est du «Rocher du Lion » à St Raphaël. Les données obtenues par l'équipe italienne de l'ENEA, en plongée avec le système de positionnement GIB, sont représentées sur cette même figure en rouge ; celles obtenues par l'IFREMER en vidéo tractée sont tracées en bordeaux avec une indication de la position des limites observées en orange. On constate que le tracé de la limité inférieure obtenu avec la méthode DIVA est cohérent avec celui des trois autres méthodes et qu'il permet de situer avec une bonne précision la frontière entre l'herbier dense et le sable.

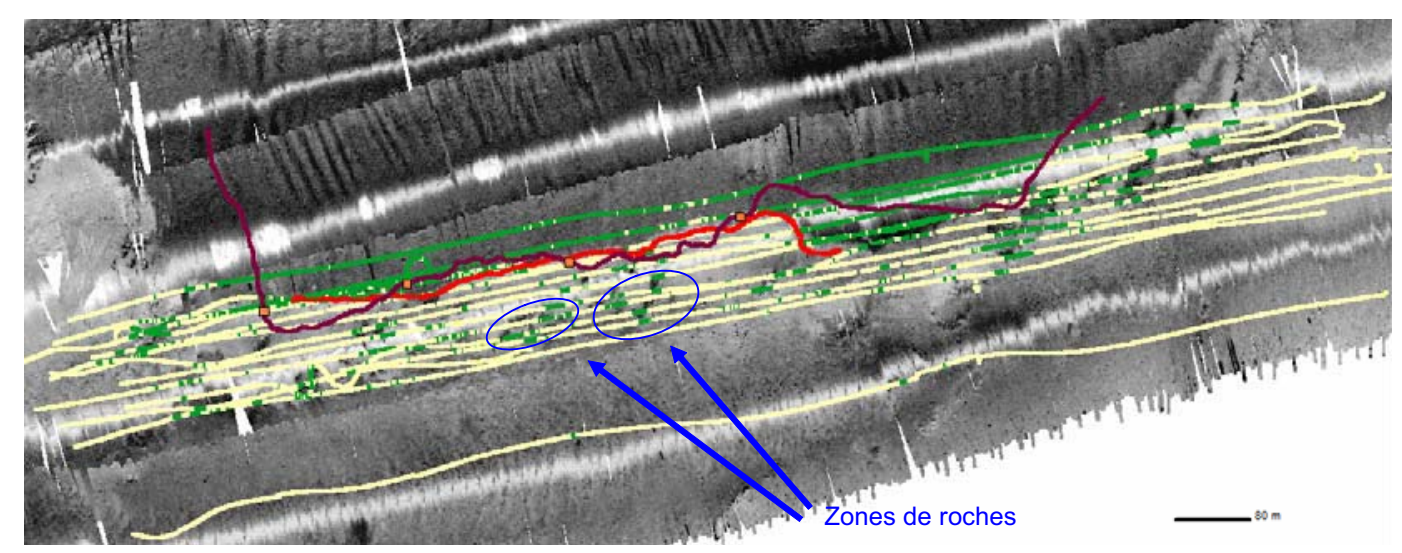

Figure 4 : Résultats de la méthode DIVA (en vert : Posidonie, en beige : sable) sur la zone A comparée aux données obtenues par plongée avec positionnement GIB (tracé rouge) et par vidéo tractée (tracé bordeau avec position des limites observées en orange).

La méthode DIVA permet de bien discriminer les faciès sable/posidonie, ce qui correspond à plus de $80 \%$ des cas : la problématique prioritaire de détection de l'herbier de plaine est donc bien résolue. Au-delà de cette limite, la méthode DIVA indique la présence de posidonies mais l'analyse de cette partie du sonogramme faite par l'IFREMER indique celle de petites zones rocheuses. Les difficultés de la méthode DIVA pour discriminer la roche de la posidonie font actuellement l'objet de développements complémentaires intégrant les faciès rocheux. Il apparaît notamment qu'un critère basé sur la largeur du signal rétrodiffusé permet, comme cela est illustré sur la figure 5 , de distinguer les petites zones de roches. 


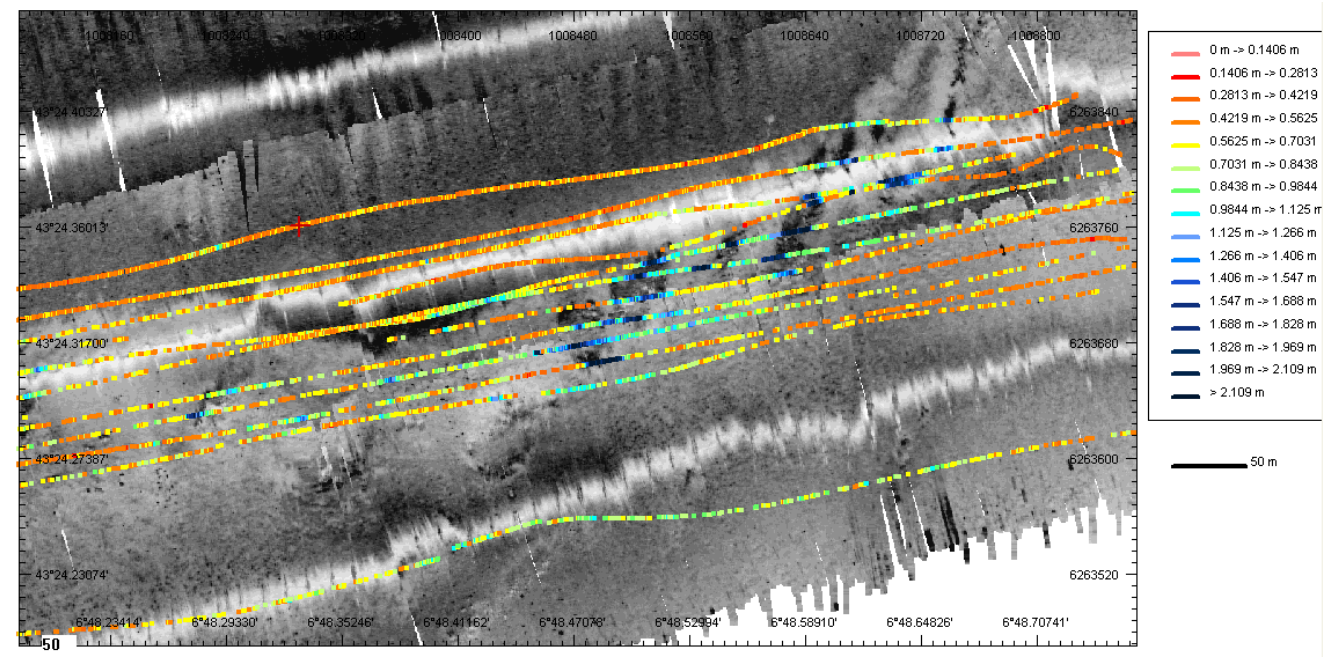

Figure 5 : Largeur du support temporel signal rétrodiffusé

La figure 6 montre les résultats de la méthode DIVA dans la baie d'Agay, superposés à la mosaïque sonal de l'IFREMER; elle met en évidence, sur des herbiers de plaine, l'excellente concordance entre la méthode DIVA et les méthodes traditionnelles.

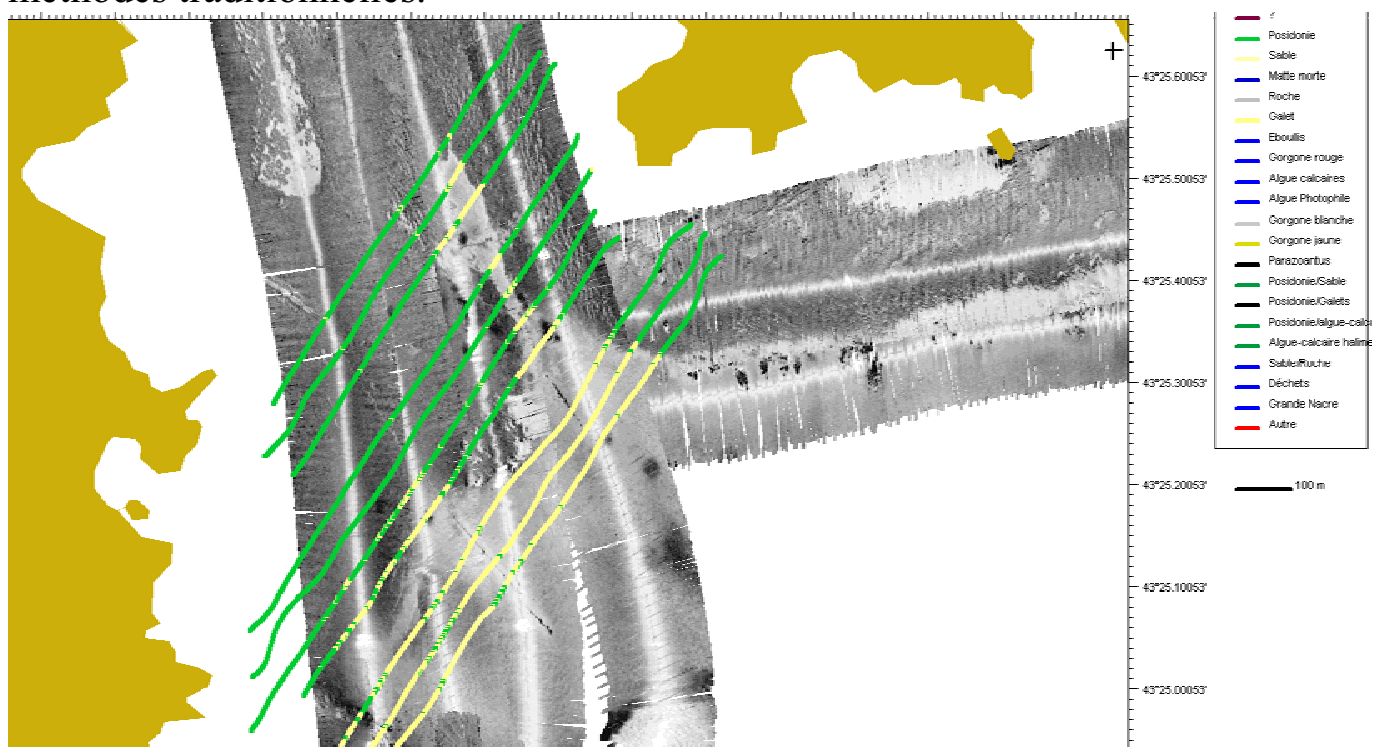

Figure 6 : Comparaison des résultats de la méthode DIVA avec la mosäque sonal en baie d'Agay (Saint Raphaël)

En l'absence de correction des mouvements de la plateforme de mesure, la mise en œuvre de la méthode DIVA est limitée à des états de mer 3 . Un système de positionnement de l'attitude du navire, actuellement à l'étude, permettra à terme de les corriger. 


\section{$4 \quad$ Conclusions}

La figure 7 synthétise les principales méthodes utilisées pour la cartographie de l'herbier de posidonie. Elle montre la complémentarité des systèmes mis en œuvre par l'IFREMER et par SEMANTIC TS.

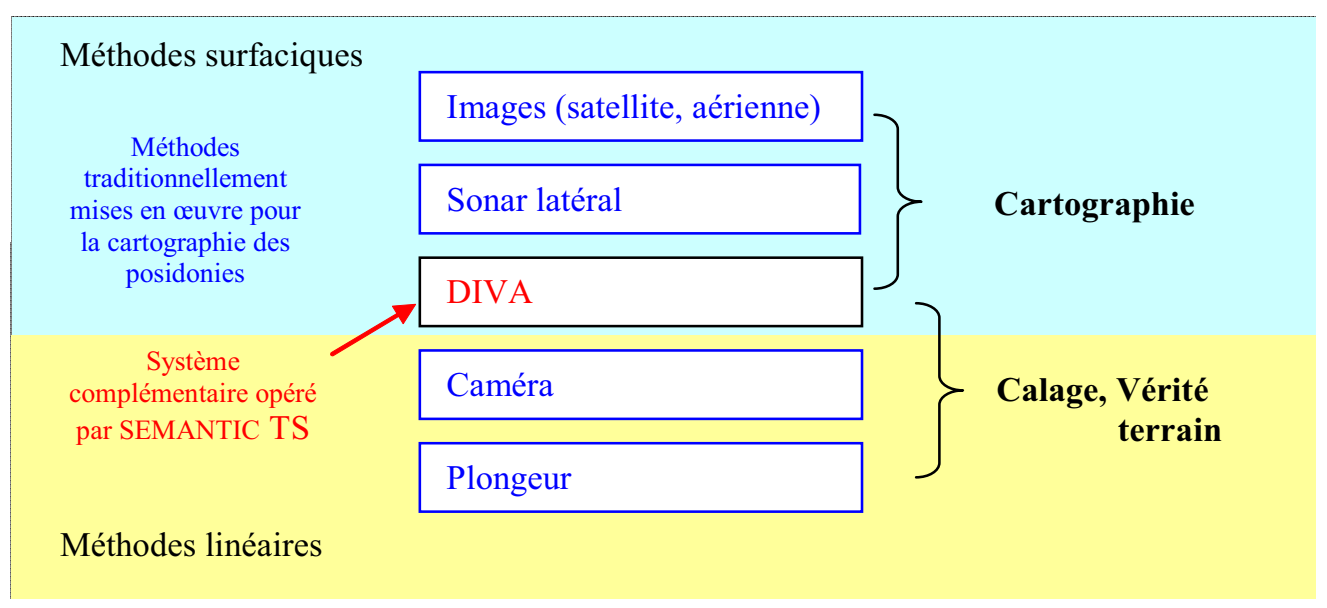

Figure 7 : Positionnement de la méthode DIVA par rapport aux méthodes traditionnelles utilisées pour la cartographie des posidonies. Compte tenu de son linéaire journalier important (100 km/jour) DIVA peut être utilisée à la fois en cartographie surfacique et en calage local.

La méthode DIVA, opérée par SEMANTIC TS, apporte des données nombreuses, précises et très bien géoréférencées, complémentaires à celles obtenues par la vidéo remorquée, qui constituent une "vérité terrain ». Elle permet de cartographier rapidement la présence/absence de végétation sur les plaines ; son linéaire journalier important $(100 \mathrm{~km}$ de radiale, par toutes profondeurs de fond) permet d'effectuer un calage des images surfaciques très résolvant et performant.

L'inconvénient principal réside dans la difficulté de reconnaissance de l'herbier sur faciès rocheux notamment.

La méthode DIVA a fourni d'excellents résultats sur des laminaires (octobre 2007) et plus récemment (juillet 2008) sur des Zostères du Bassin d'Arcachon.

Par ailleurs, compte tenu de sa grande précision dans le géoréférencement (inférieure au mètre), la méthode DIVA permet aisément d'effectuer un suivi dans le temps de l'évolution de l'herbier. 
En outre, compte tenu de l'important linéaire journalier réalisable (une centaine de kilomètres si les conditions météo-océaniques le permettent), la méthode DIVA peut aussi être considérée comme un moyen de cartographie peu onéreux lorsqu'il est utilisé seul. Le pouvoir couvrant de cette méthode est certes faible, mais elle permet - en multipliant le nombre de radiales - de détecter et de délimiter rapidement la limite inférieure de l'herbier de posidonie. Elle permet également d'effectuer des interventions localisées sur des zones de doute.

En ce sens, la méthode DIVA permet de se substituer, selon la nature et l'étendue du travail cartographique à effectuer, à d'autres méthodes plus précises mais plus lentes (plongées, vidéo remorquée) ou plus rapides mais moins précises (sonar).

Les méthodes mises en œuvre par l'IFREMER (images aériennes ou satellitales, sonal) permettent d'obtenir une cartographie surfacique importante et sont indispensables pour une appréciation à grande échelle de la couverture de l'herbier. Leur validation peut être effectuée par des techniques des plongées, de la vidéo remorquée, ou la méthode DIVA suivant les besoins.

\section{$5 \quad$ Références bibliographiques}

1 LURTON X (2002) An introduction to underwater acoustics: principles and applications. Edition Springer - ISBN 3-540-42967-0, 2002

2 NOEL C, VIALA C, LEHN E, JAUFFRET C (2005) Développement d'une méthode acoustique de détection des herbiers de posidonies. Colloque : Sciences et technologies marines du futur - Un enjeu pour la Méditerranée. Marseille Hotel de Région - 19-20 Mai 2005

3 VIALA C, NOEL C, ZERR B (2006) Acoustic characterization of underwater vegetations. Colloque Caractérisation du Milieu Marin, Brest 16-19 oct 2006.

4 VIALA C, NOEL C, COQUET M, ZERR B, LELONG P, BONNEFONT JL (2006) Pertinence de la méthode DIVA pour l'interprétation des mosaïques sonar latéral. 3rd Mediterranean Symposium on Marine Vegetation. Marseille, 03/ 2007.

5 VIALA C, NOEL C, COQUET M, ZERR B, PERROT T. Acoustic data fusion devoted to underwater vegetation mapping. ECUA/SFA Paris, 28/06 - 04/07 2008

6 GUYOMARD P (1994) Cartographie de données sédimentologiques acquises au large de Tréport. Rapport EPSHOM.

7 SHOM (1997) Fiche de synthèse opérationnelle thématique, les sédiments marins. Documentation opérationnelle, 732BXOM, EPSHOM.

\section{$6 \quad$ Remerciements}

Les auteurs remercient l'Agence de l'Eau Rhône Méditerranée et Corse ainsi que la Région PACA qui ont soutenu financièrement ces travaux. 